\title{
Determination of interbilayer and transbilayer lipid transfers by time- resolved small-angle neutron scattering
}

\section{AUTHOR(S):}

Nakano, M; Fukuda, M; Kudo, T; Endo, H; Handa, T

\section{CITATION:}

Nakano, M ...[et al]. Determination of interbilayer and transbilayer lipid transfers by timeresolved small-angle neutron scattering. PHYSICAL REVIEW LETTERS 2007, 98(23): 238101.

\section{ISSUE DATE:}

2007-06-08

URL:

http://hdl.handle.net/2433/50062

RIGHT:

Copyright 2007 American Physical Society 


\title{
Determination of Interbilayer and Transbilayer Lipid Transfers by Time-Resolved Small-Angle Neutron Scattering
}

\author{
Minoru Nakano, ${ }^{1, *}$ Masakazu Fukuda, ${ }^{1}$ Takayuki Kudo, ${ }^{1}$ Hitoshi Endo, ${ }^{2}$ and Tetsurou Handa ${ }^{1}$ \\ ${ }^{1}$ Graduate School of Pharmaceutical Sciences, Kyoto University, Kyoto 606-8501, Japan \\ ${ }^{2}$ Institute for Solid State Physics, The University of Tokyo, Tokai, 319-1106, Japan
}

(Received 9 February 2007; published 7 June 2007)

\begin{abstract}
We applied a time-resolved small-angle neutron scattering technique to the vesicle system of dimyristoylphosphatidylcholine for the first time to determine lipid kinetics. The observed kinetics could be explicitly represented by a simple model that includes two independent kinetic parameters, i.e., the rates of transbilayer and interbilayer exchange. This technique is perfectly suited for the determination of lipid exchange kinetics in equilibrium and applicable to evaluation of the activity of the factors relevant to lipid migration, such as translocase and lipid transfer proteins.
\end{abstract}

DOI: 10.1103/PhysRevLett.98.238101

PACS numbers: 87.16.Dg, 87.14.Cc, 87.64.Bx

In biological plasma membranes, interbilayer transport and transbilayer movement of phospholipids are controlled by lipid transfer proteins and translocase enzymes [1-4]. For example, phosphatidylinositol (PI)-transfer proteins bind PI and phosphatidylcholine (PC) and function as PIPC exchange proteins, whose function is required for receptor-mediated signaling of phosphatidylinositol-4,5bisphosphate hydrolysis [5] and vesiculating activity from the trans-Golgi network membrane [6]. The endoplasmic reticulum, in which phospholipids are newly synthesized on the cytosolic leaflet, maintains membrane symmetry, presumably by flippase activity [7], which is bidirectional and energy independent [8,9]. On the other hand, the plasma membrane retains asymmetric lipid distribution by aminophospholipid translocase that mediates the unidirectional transport of phosphatidylserine and phosphatidylethanolamine from cytoplasmic to ectoplasmic leaflets of the bilayer [10]. Disruption of the asymmetry in cells is involved in apoptosis and associated with increased binding and phagocytosis of these cells by macrophage [11].

Evaluation of these lipid dynamics is, however, limited due to its methodological difficulties. Fluorescence spectroscopy [12-15] and electron spin resonance $[16,17]$ are most widely used for investigating lipid flip-flop. These experiments require the introduction of fluorescence or spin labels into the lipids which may change their physical and chemical properties [18]. The use of isotopes [18-23] avoids artifacts of the labeling. The rates of transbilayer and interbilayer exchange of ${ }^{3} \mathrm{H}$-labeled dimyristoylphosphatidylcholine (DMPC) have been examined in small unilamellar vesicles (SUV's) [20] and large unilamellar vesicles (LUV's) [22,23]. In this method, however, detection of the transfer of ${ }^{3} \mathrm{H}$-DMPC from donor to acceptor vesicles requires differences in charge [20,22] or size [23] to separate the two vesicle types on columns and a nonexchangeable marker to correct for donor recovery. A baseline to estimate the nonexchangeable fraction is an additional fitting parameter, which may influence the determination of the exchange rates.

Time-resolved small-angle neutron-scattering (TRSANS) technique has been used to determine self-diffusion coefficients in polymer melts [24,25]. Richter and coworkers [26-28] have reported that the unimer exchange of polymer micelles in dimethylformamide and in water/ dimethylformamide mixture can be detected by TR-SANS technique using hydrogenated and deuterated polymers. This is based on the fact that scattering length densities (SLD's) are significantly different between micelles consisting of hydrogenated and deuterated polymers, and that the unimer exchange between these micelles reduces the difference in SLD with time. This technique can be applied to phospholipids since the transfer of lipids is known to be a slow process with a half-life of hours, i.e., detectable time scale by SANS.

Here, we describe TR-SANS measurements on mixtures of LUV's consisting of hydrogenated and deuterated DMPC as the first application to the lipid vesicle system, and demonstrate that TR-SANS is a powerful tool to investigate membrane lipid dynamics so that both interbilayer and transbilayer lipid transfers can be simultaneously and unambiguously determined.

LUV's were prepared by the extrusion method [29] using a polycarbonate membrane with a pore size of $100 \mathrm{~nm}$. The solvent used in this study was Tris-buffered saline containing an equivalent volume of $\mathrm{D}_{2} \mathrm{O}$ and $\mathrm{H}_{2} \mathrm{O}$ (10 $\mathrm{m} M$ Tris, $150 \mathrm{~m} M \mathrm{NaCl}, 1 \mathrm{~m} M$ EDTA, and $0.01 \%$ $\mathrm{NaN}_{3}, p H$ 7.4). In addition to LUV's consisting of either $d_{54}$-DMPC (D-LUV) or DMPC (H-LUV), LUV's consisting of a 1:1 mixture of both lipids (D/H-LUV) were prepared by mixing these lipids before hydration. The phospholipid concentration of each LUV preparation was set to $20 \mathrm{~m} M$. SANS measurements were performed by SANS-U of the Institute for Solid State Physics, the University of Tokyo, at the research reactor JRR-3, Tokai, Japan [30]. The wavelength $(\lambda)$ of neutron source 
was $7 \AA(\Delta \lambda / \lambda=10 \%)$. The sample-to-detector distance was set to $4 \mathrm{~m}$ to cover a $q$ range of $0.007 \leq q \leq 0.1 \AA^{-1}$. Samples were measured in quartz cells with a pass length of $2 \mathrm{~mm}$. Scattering data measured by the 2D-detector were corrected for electronic background and circular averaged to be $1 \mathrm{D}$-form scattering data. The scattering of the empty cell was subtracted. The data were transformed to absolute cross sections using a Lupolen $®$ standard and subtracted for solvent scattering. TR-SANS experiments were carried out at four different temperatures. Each measurement was started immediately after mixing an equivalent volume of D-LUV and H-LUV. Data were accumulated every $3 \mathrm{~min}$.

The SLD's of DMPC and $d_{54}$-DMPC were calculated as $0.28 \times 10^{10}$ and $5.40 \times 10^{10} \mathrm{~cm}^{-1}$, respectively, using a reported partial volume of $0.975 \mathrm{~cm}^{3} / \mathrm{g}$ [31]. As shown in Fig. 1(a), the average of these SLD's $\left(2.84 \times 10^{10} \mathrm{~cm}^{-1}\right)$ corresponds to the SLD of water with 50 vol \% $\mathrm{D}_{2} \mathrm{O}$ $\left(2.92 \times 10^{10} \mathrm{~cm}^{-1}\right)$. Thus, we used Tris buffer with 50 vol $\% \mathrm{D}_{2} \mathrm{O}$ as a solvent in this study. SANS profiles of LUV's consisting of either $d_{54}$-DMPC (D-LUV) or DMPC (H-LUV) are shown in Fig. 2(a). D-LUV and H-LUV showed identical scattering profiles. The profiles were nearly proportional to $q^{-2}$, where $q$ is the scattering vector $[=4 \pi(\sin \theta) / \lambda]$. This is a typical behavior of planar structures and reflects the shell construction of LUV. It should be noted that the spherical feature of LUV appears in profile of much lower $q$ regions. As opposed to the above two LUV's, D/H-LUV exhibited little scattering, suggesting that the SLD of D/H-LUV accords with that of the

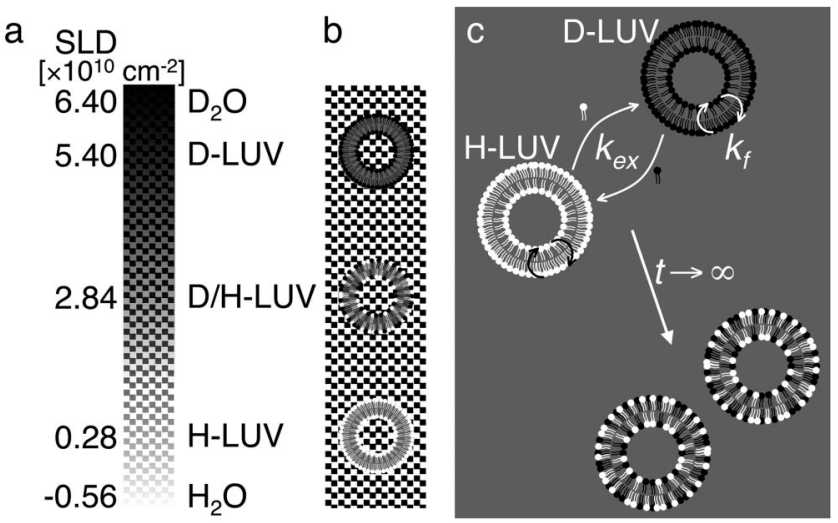

FIG. 1. Principle of the detection of lipid dynamics by TRSANS. (a) Calculated SLD's of LUV's and solvents are graded, where high and low SLD are represented by black and white, respectively. SLD of D/H-LUV corresponds to that of water with 50 vol $\% \mathrm{D}_{2} \mathrm{O}$ (contrast matching). (b) In $50 \mathrm{vol} \% \mathrm{D}_{2} \mathrm{O}$, DLUV (top), and H-LUV (bottom) are "visible," while D/H-LUV (middle) is invisible by neutrons under contrast-matching conditions. (c) Lipid exchange between D- and H-LUV and flip-flop in the membranes reduces the contrast of LUV's with solvent and LUV's become less visible, which can be detected as a decrease in the scattering intensity by TR-SANS experiment. buffer, i.e., D/H-LUV is "invisible" for neutrons in this contrast-matching condition [Fig. 1(b)].

When equimolar amounts of D-LUV and H-LUV are mixed, scattering intensity should be equal to the average of each LUV preparation if there is no intervesicular lipid exchange. On the other hand, the lipid exchange between D- and H-LUV reduces the difference in the SLD of LUV's from solvent (i.e., contrast) with time as schematically shown in Fig. 1(c). This leads to a decrease in the scattering intensity. As shown in Fig. 2(b), the scattering from a mixture of D- and H-LUV faded over time, suggesting lipid exchange in the observational time scale. In addition, at all times, the SANS profile maintained almost an identical shape. This indicates that the shell construction of LUV is maintained during the lipid exchange process. In this case, the intensity change depends only on the contrast. The normalized contrast, $\Delta \rho(t) / \Delta \rho(0)$, is given by $(\sqrt{I(t)}-\sqrt{I(\infty))} /(\sqrt{I(0)}-\sqrt{I(\infty))}$, where $I(t)$ is the scattering intensity at time $t$ after LUV mixing. Here, we took integral intensity in the $q$ region as between 0.007 and $0.03 \AA^{-1}$. The residual intensity at infinite time, $I(\infty)$, and initial intensity, $I(0)$, correspond to the intensity of $\mathrm{D} / \mathrm{H}-\mathrm{LUV}$ and the average intensity of D- and H-LUV, respectively, which can be determined from Fig. 2(a). Time courses of the normalized contrast at four different temperatures are shown in Fig. 3. The contrast decayed more steeply with an increase in temperature. In addition, the normalized contrasts reached well below 0.5 , suggesting the involvement of flip-flop. The kinetics of the lipid exchange mechanism illustrated in Fig. 1 is described by the following differential equations with rate constants of the exchange $\left(k_{\mathrm{ex}}\right)$ and flip-flop $\left(k_{f}\right)$ :

$$
-\frac{d\left|\Delta \rho_{\text {out }}\right|}{d t}=k_{\text {ex }}\left(\left|\Delta \rho_{\text {out }}\right|-0\right)+k_{f}\left(\left|\Delta \rho_{\text {out }}\right|-\left|\Delta \rho_{\text {in }}\right|\right),
$$
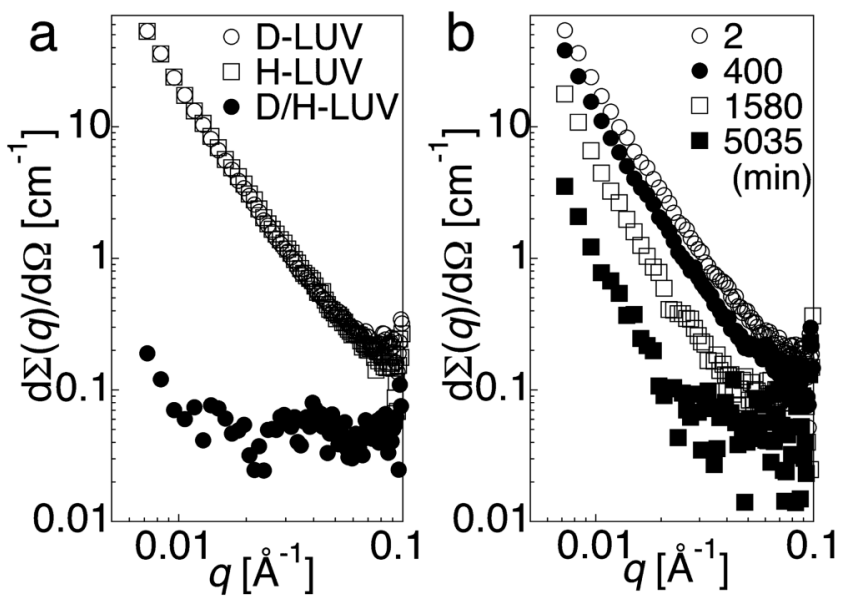

FIG. 2. (a) SANS profiles of D-LUV, H-LUV, and D/H-LUV in buffer with $50 \% \mathrm{D}_{2} \mathrm{O}$ at $27.1^{\circ} \mathrm{C}$. (b) SANS profiles of $1: 1$ mixture of D- and H-LUV 2, 400, 1580, and 5035 min after mixing at $27.1^{\circ} \mathrm{C}$. 


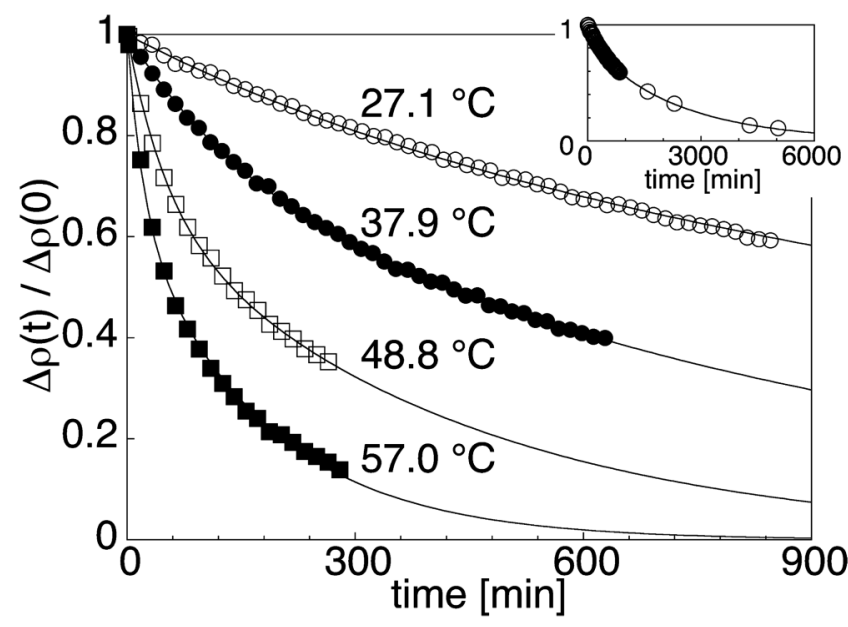

FIG. 3. Contrast decays of LUV's after mixing D- and H-LUV at four different temperatures. Solid curves are fitting curves according to Eq. (5). Inset shows the contrast decay over a longer period at $27.1^{\circ} \mathrm{C}$.

$$
-\frac{d\left|\Delta \rho_{\text {in }}\right|}{d t}=-k_{f}\left(\left|\Delta \rho_{\text {out }}\right|-\left|\Delta \rho_{\text {in }}\right|\right),
$$

where, $\Delta \rho_{\text {in }}$ and $\Delta \rho_{\text {out }}$ are the contrasts of inner and outer leaflets of LUV with solvent, respectively. D- and H-LUV can be assumed to have identical absolute values of contrast where one is positive and the other is negative. The term of -0 in Eq. (1) denotes that back exchange from DLUV and H-LUV equally takes place. With an initial condition of $\Delta \rho_{\text {in }}(0)=\Delta \rho_{\text {out }}(0)=1$, simultaneous differential equations [Eqs. (1) and (2)] can be solved:

$$
\begin{aligned}
\left|\Delta \rho_{\text {out }}\right|= & \frac{k_{\mathrm{ex}}-k_{2}}{k_{1}-k_{2}} \exp \left(-k_{1} t\right)+\frac{k_{1}-k_{\mathrm{ex}}}{k_{1}-k_{2}} \exp \left(-k_{2} t\right), \\
\left|\Delta \rho_{\mathrm{in}}\right|= & \frac{k_{\mathrm{ex}}-k_{2}}{k_{1}-k_{2}} \frac{k_{f}+k_{\mathrm{ex}}-k_{1}}{k_{f}} \exp \left(-k_{1} t\right) \\
& +\frac{k_{1}-k_{\mathrm{ex}}}{k_{1}-k_{2}} \frac{k_{f}+k_{\mathrm{ex}}-k_{2}}{k_{f}} \exp \left(-k_{2} t\right),
\end{aligned}
$$

where, $k_{1}=\left(k_{\mathrm{ex}}+2 k_{f}+\sqrt{4 k_{f}^{2}+k_{\mathrm{ex}}^{2}}\right) / 2$ and $k_{2}=\left(k_{\mathrm{ex}}+\right.$ $\left.2 k_{f}-\sqrt{4 k_{f}^{2}+k_{\mathrm{ex}}^{2}}\right) / 2$. The normalized contrast, $\Delta \rho(t) / \Delta \rho(0)$, is an average of $\left|\Delta \rho_{\text {in }}\right|$ and $\left|\Delta \rho_{\text {out }}\right|$, and is finally represented by a double-exponential decay function,

$$
\begin{aligned}
\frac{\Delta \rho(t)}{\Delta \rho(0)}= & \left(\frac{1}{2}-\frac{k_{f}}{X}\right) \exp \left(-\frac{k_{\mathrm{ex}}+2 k_{f}+X}{2} t\right) \\
& +\left(\frac{1}{2}+\frac{k_{f}}{X}\right) \exp \left(-\frac{k_{\mathrm{ex}}+2 k_{f}-X}{2} t\right),
\end{aligned}
$$

where, $X=\sqrt{4 k_{f}^{2}+k_{\mathrm{ex}}^{2}}$. The obtained contrast decays could not be fitted by a single-exponential function, and were well reproduced by Eq. (5), as represented by solid curves in Fig. 3. Arrhenius plots of the obtained parameters exhibited a good linear relationship (Fig. 4). The half-lives $\left(t_{1 / 2}=(\ln 2) / k\right)$ of the lipid exchange at 50 and $30^{\circ} \mathrm{C}$ were estimated at 0.67 and $5.4 \mathrm{~h}$, respectively, which are close to or slightly smaller than those obtained using a radioisotope (0.74 and $9.6 \mathrm{~h}$, respectively) [22]. Thermodynamic parameters at $37.0^{\circ} \mathrm{C}$ were calculated from the plot using the method described in Homan and Pownall [32], and are listed in Table I. Both lipid exchange and flip-flop are associated with an increase in enthalpy and a decrease in entropy of the transition-state complex formation. It is interesting that while the values of the free energy of activation are similar, the flip-flop has reduced values of activation enthalpy and entropy compared with the interbilayer exchange. This result suggests that an entropically more unfavorable process is involved in the transition state of flip-flop, which needs to be elucidated.

In summary, we succeeded in the determination of the kinetics of transfer for lipids that migrate between leaflets of the bilayer and traverse the aqueous phase separating vesicles. This method involves the simple process of mixing deuterated and hydrogenated compounds for TRSANS measurement, and no complicated operation is required. Although a number of complex kinetic models have been proposed [33,34], the kinetics can be described by a very simple equation [Eq. (5)] in the system we used. Evaluation of the TR-SANS data using only two independent kinetic parameters enables us to determine these parameters unambiguously. In addition, the flip-flop of lipids before sample mixing has no influence, which is strictly distinct from methods using asymmetrically labeled bilayers, where immediate measurement is required after sample preparation [13,18]. Also, since the influence of additives such as proteins and peptides on neutron scattering can be ignored at a considerably lower concentration than lipids, TR-SANS can be applied to the systems of proteoliposomes or liposomes in the presence of

TABLE I. Thermodynamic parameters of DMPC exchange and flip-flop at $37.0^{\circ} \mathrm{C}$.

\begin{tabular}{lcccccc}
\hline \hline & $E_{a}{ }^{\mathrm{a}}(\mathrm{kJ} / \mathrm{mol})$ & $k^{\mathrm{b}}\left(\mathrm{min}^{-1}\right)$ & $t_{1 / 2}{ }^{\mathrm{b}}(\min )$ & $\Delta G^{\ddagger \mathrm{c}}(\mathrm{kJ} / \mathrm{mol})$ & $\Delta H^{\ddagger \mathrm{c}}(\mathrm{kJ} / \mathrm{mol})$ & $T \Delta S^{\ddagger \mathrm{c}}(\mathrm{kJ} / \mathrm{mol})$ \\
\hline Exchange & 84.6 & $4.60 \times 10^{-3}$ & 151 & 101 & 82.1 & -18.4 \\
Flip-flop & 64.0 & $1.35 \times 10^{-3}$ & 513 & 104 & 61.5 & -42.2 \\
\hline \hline
\end{tabular}

${ }^{\mathrm{a}}$ Activation energies $\left(E_{a}\right)$ of exchange and flip-flop have experimental errors of $2.8 \%$ and $8.1 \%$, respectively.

${ }^{\mathrm{b}}$ The rate constants $(k)$ and half-lives $\left(t_{1 / 2}\right)$ of exchange and flip-flop are extrapolated to $37.0^{\circ} \mathrm{C}$ from the Arrhenius data.

${ }^{\mathrm{c}}$ Thermodynamic parameters of the activated states were calculated with the method described in Homan and Pownall [32]. 


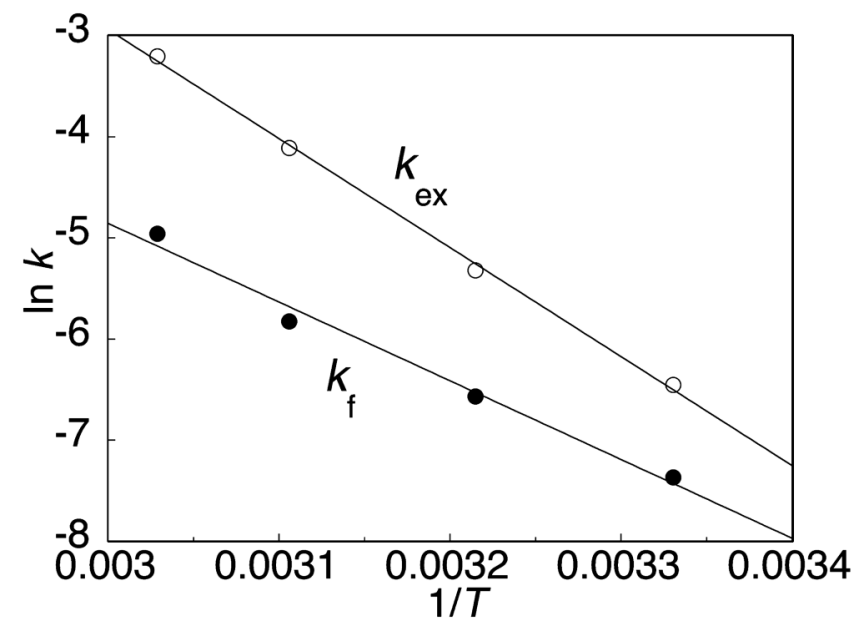

FIG. 4. Arrhenius plots of the rates of intervesicular exchange $\left(k_{\mathrm{ex}}\right)$ and flip-flop $\left(k_{f}\right)$.

soluble proteins. Although the determination of the flipflop rate by TR-SANS is, similar to the method using radioisotopes [22,23] and fluorophores [35], limited to systems that involve faster exchange than the flip-flop, it can be concluded that TR-SANS is a powerful tool to evaluate the activity of translocase and lipid transfer proteins.

This work was carried out by joint research of the Institute for Solid State Physics, the University of Tokyo (Proposal No. 6545). This study was supported by Grantsin-aid for Scientific Research from the Japanese Ministry of Education, Culture, Sports, Science and Technology (No. 17390011 and No. 17655005), Foundation of Advanced Technology Institute, and the program for the Promotion of Fundamental Studies in Health Science of the National Institute of Biomedical Innovation (NIBIO).

*Corresponding author.

Electronic address: mnakano@pharm.kyoto-u.ac.jp

[1] T. R. Graham, Trends Cell Biol. 14, 670 (2004).

[2] J. C. M. Holthuis and T.P. Levine, Nat. Rev. Mol. Cell Biol. 6, 209 (2005).

[3] M. A. Kol, A. I. P. M. de Kroon, J.A. Killian, and B. de Kruijff, Biochemistry 43, 2673 (2004).

[4] T. Pomorski, J. C. M. Holthuis, A. Herrmann, and G. van Meer, J. Cell Sci. 117, 805 (2004).

[5] A. Kauffmannzeh, G. M. H. Thomas, A. Ball, S. Prosser, E. Cunningham, S. Cockcroft, and J. J. Hsuan, Science 268, 1188 (1995).

[6] J. P. Simon, T. Morimoto, V. A. Bankaitis, T. A. Gottlieb, I. E. Ivanov, M. Adesnik, and D. D. Sabatini, Proc. Natl. Acad. Sci. U.S.A. 95, 11181 (1998).

[7] M.S. Bretsche, Science 181, 622 (1973).

[8] W. R. Bishop and R. M. Bell, Cell 42, 51 (1985).
[9] X. Buton, G. Morrot, P. Fellmann, and M. Seigneuret, J. Biol. Chem. 271, 6651 (1996).

[10] M. Seigneuret and P.F. Devaux, Proc. Natl. Acad. Sci. U.S.A. 81, 3751 (1984).

[11] L. Mcevoy, P. Williamson, and R. A. Schlegel, Proc. Natl. Acad. Sci. U.S.A. 83, 3311 (1986).

[12] S. Hrafnsdottir, J.W. Nichols, and A.K. Menon, Biochemistry 36, 4969 (1997).

[13] M. A. Kol, A. N.C. van Laak, D. T. S. Rijkers, J. A. Killian, A.I.P.M. de Kroon, and B. de Kruijff, Biochemistry 42, 231 (2003).

[14] J. Kubelt, A. K. Menon, P. Muller, and A. Herrmann, Biochemistry 41, 5605 (2002).

[15] U. Marx, G. Lassmann, H. G. Holzhutter, D. Wustner, P. Muller, A. Hohlig, J. Kubelt, and A. Herrmann, Biophys. J. 78, 2628 (2000).

[16] R. D. Kornberg and H. M. Mcconnel, Biochemistry 10, 1111 (1971).

[17] M. G. Mcnamee and H. M. Mcconnel, Biochemistry 12, 2951 (1973).

[18] J. Liu and J. C. Conboy, Biophys. J. 89, 2522 (2005).

[19] B. De Kruijff and E. J. J. Van Zoelen, Biochim. Biophys. Acta 511, 105 (1978).

[20] L. R. Mclean and M. C. Phillips, Biochemistry 23, 4624 (1984).

[21] H. M. Reinl and T. M. Bayerl, Biochemistry 33, 14091 (1994).

[22] W. C. Wimley and T. E. Thompson, Biochemistry 29, 1296 (1990).

[23] W. C. Wimley and T. E. Thompson, Biochemistry 30, 1702 (1991).

[24] C. R. Bartels, B. Crist, and W.W. Graessley, Macromolecules 17, 2702 (1984).

[25] C. R. Bartels, W. W. Graessley, and B. Crist, J. Polym. Sci., Polym. Lett. Ed. 21, 495 (1983).

[26] R. Lund, L. Willner, D. Richter, and E. E. Dormidontova, Macromolecules 39, 4566 (2006).

[27] R. Lund, L. Willner, J. Stellbrink, P. Lindner, and D. Richter, Phys. Rev. Lett. 96068302 (2006).

[28] L. Willner, A. Poppe, J. Allgaier, M. Monkenbusch, and D. Richter, Europhys. Lett. 55, 667 (2001).

[29] R. C. Macdonald, R. I. Macdonald, B. P. M. Menco, K. Takeshita, N. K. Subbarao, and L. R. Hu, Biochim. Biophys. Acta 1061, 297 (1991).

[30] S. Okabe, M. Nagao, T. Karino, S. Watanabe, T. Adachi, H. Shimizu, and M. Shibayama, J. Appl. Crystallogr. 38, 1035 (2005).

[31] P. Westh and C. Trandum, J. Phys. Chem. B 104, 11334 (2000).

[32] R. Homan and H. J. Pownall, Biochim. Biophys. Acta 938, 155 (1988).

[33] T. Arvinte and K. Hildenbrand, Biochim. Biophys. Acta 775, 86 (1984).

[34] J. Storch and A. M. Kleinfeld, Biochemistry 25, 1717 (1986).

[35] J.N. Bai and R.E. Pagano, Biochemistry 36, 8840 (1997). 